
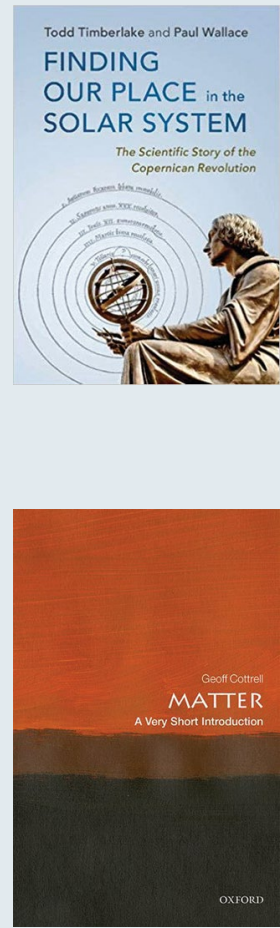

\section{Matter: A Very Short Introduction}

By Geoff Cottrell OXFORD UNIVERSITY PRESS: 2019. 176PP. $€ 8.99$

In the Very Short Introduction series of booklets, Geoff Cottrell adds Matter, a condensed but all-encompassing introduction to what matter is. From the smallest scales (quantum structures) to the largest (clusters of galaxies and beyond), the book goes over the laws of nature that dictate how matter forms, aggregates together and creates observable effects. In the course of this introduction, quantum laws, thermodynamics and general relativity all figure prominently. The last third of the book is focused on the astrophysical aspects of matter, starting from the Big Bang and nucleosynthesis to dark matter and dark energy.

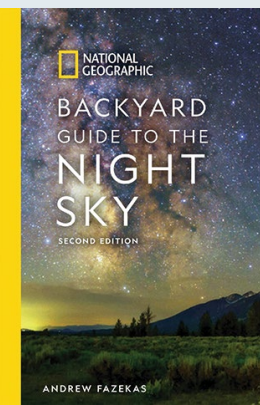

\section{Backyard Guide to the Night Sky}

By Andrew Fazekas

NATIONAL GEOGRAPHIC: 2019. 288PP. £19.03

Under the unassuming name Backyard Guide to the Night Sky, Andrew Fazekas actually compiles a thorough and well-illustrated introduction to observational astronomy oriented towards amateur astronomers or simple enthusiasts. The book focuses more on the phenomenology of some of the key sky players (ranging from the Sun, to other planetary bodies, our Galaxy and beyond). The book also includes chapters on the kind of instruments and tools one might use to observe the night sky from their backyard (binoculars, telescopes and so on), as well as sky charts organized according to season and the constellations on the sky.

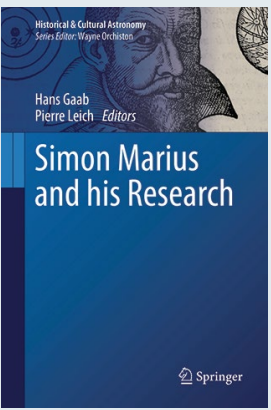

\section{Simon Marius and his Research}

Edited by Hans Gaab and Pierre Leich SPRINGER INTERNATIONAL PUBLISHING: 2019. XII, 481PP. $€ 109.99$

Simon Marius was a German astronomer that lived and worked in the 16th century whose research was right at the boundary between the Earth-centric and heliocentric systems. He used the contemporarily developed telescope to observe Jupiter and discover four of its moons (independently from Galileo) but also worked on the Sun, comets and other topics of interest at that time (including the making of calendars and astrology). This tome provides a comprehensive overview of Marius's life, historical environment and research, offering an interesting alternative and largely overlooked viewpoint of the scientific revolution brought about by Copernicus.

Published online: 9 May 2019

https://doi.org/10.1038/s41550-019-0778-z 